\title{
Factors influencing protective behaviours during haze episodes in Singapore: A population-based study
}

Kennedy YY Ng ${ }^{* 1}{ }_{M B B S}$, Wesley Yeung ${ }^{* 2}{ }_{M B B S}$, Ka Lon $\underline{\operatorname{Sou}}{ }^{* 3}{ }_{P h D}$, Jie Xin $\underline{\operatorname{Lim}}{ }^{* 4}{ }_{P h D}$, Sai Liang ${ }^{5}{ }_{M B B S}$, Ryan KJ $\underline{\text { Lee }}{ }^{6 B B B S}$, Nigel JM Fong ${ }^{7} M B B S$, Alex Lua ${ }^{8}$ MBBS, Xinqi Look ${ }^{9}{ }_{M B B S}$, Julia Ann-Lee ${ }^{10}{ }_{M B B S}$, Yun Hao Leong ${ }^{11}{ }_{M B B S}$, Claudia $\underline{\text { Chong }}{ }^{12} M B B S$, Kai Yun $\underline{\text { Ang }}{ }^{13}{ }_{M B B S}$, Cheryl Lie ${ }^{14}{ }_{M B B S}$, Amanda $\underline{\text { Chin }}{ }^{15}{ }_{M B B S}$, Judy Gek Khim $\underline{\text { Sng }}{ }^{16}{ }_{M B B S}$, Bee Choo Tai ${ }^{16}{ }_{P h D}$

\begin{abstract}
Introduction: Haze is a recurrent problem in Southeast Asia. Exposure to haze is linked to ophthalmic, respiratory and cardiovascular diseases, and mortality. In this study, we investigated the role of demographic factors, knowledge and perceived risk in influencing protective behaviours during the 2013 haze in Singapore.

Methods: We evaluated 696 adults in a cross-sectional study. Participants were sampled via a 2-stage simple random sampling without replacement from a large residential district in Singapore in 2015. The questionnaire measured the participant's knowledge, perceived risk and behaviours during the Southeast Asian haze crisis in 2013. Reliability and validity of the questionnaire were assessed using comparative fit index $(\geq 0.96)$ and root mean square error of approximation $(\leq 0.05)$. We performed structural equation modelling to examine the relationship between the hypothesised factors and protective behaviours.

Results: More than $95 \%$ of the individuals engaged in at least 1 form of protective behaviour. Knowledge was strongly associated with protective behaviours via direct effect $(\beta=0.45,95 \%$ CI $0.19-0.69, P<0.001)$ and indirect effect through perceived risk $(\beta=0.18,95 \%$ CI $0.07-0.31, P=0.002)$. Perceived risk was associated with protective behaviours $(\beta=0.28,95 \% \mathrm{CI}: 0.11-0.44, P=0.002)$. A lower household income and ethnic minority were associated with protective behaviours. A lower education level and smokers were associated with lower knowledge of haze. A higher education and ethnic minority were associated with a lower perceived risk. Wearing of N95 masks was associated with other haze-related protective behaviours $(\beta=0.24,95 \%$ CI $0.08-0.37, P=0.001)$.

Conclusion: Knowledge was associated with protective behaviours, suggesting the importance of public education. Efforts should target those of lower education level and smokers. The wearing of N95 masks correlates with uptake of other protective behaviours.
\end{abstract}

Ann Acad Med Singap 2021;50:514-26

Keywords: Haze, knowledge, N95 mask, protective behaviour, risk perception

\footnotetext{
${ }^{1}$ Division of Medical Oncology, National Cancer Centre Singapore, Singapore

${ }^{2}$ University Medicine Cluster, National University Hospital, Singapore

${ }^{3}$ Singapore University of Technology and Design, Singapore

${ }^{4}$ Division of Psychology, School of Social Sciences, Nanyang Technological University, Singapore

${ }^{5}$ Department of Neurosurgery, National Neuroscience Institute, Singapore

${ }^{6}$ Yong Loo Lin School of Medicine, National University of Singapore, Singapore

${ }^{7}$ Division of Medicine, Singapore General Hospital, Singapore

${ }^{8}$ Department of Urology, Tan Tock Seng Hospital, Singapore

${ }^{9}$ Post-acute and Continuing Care, Outram Community Hospital, Singapore

${ }^{10}$ Division of Family Medicine, National University Hospital, Singapore

${ }^{11}$ Department of Anaesthesia, Singapore General Hospital, Singapore

${ }^{12}$ Department of Anaesthesia, Tan Tock Seng Hospital, Singapore

${ }^{13}$ Department of Anaesthesia, National University Hospital, Singapore

${ }^{14}$ Dermatology Service, KK Women's and Children's Hospital, Singapore

${ }^{15}$ Division of Neurology, Department of Medicine, National University Hospital, Singapore

${ }^{16}$ Saw Swee Hock School of Public Health, National University of Singapore, Singapore

Correspondence: Dr Kennedy Yao Yi Ng, Division of Medical Oncology, National Cancer Centre Singapore, 11 Hospital Crescent, Singapore 169610.

Email: kennedy.ng.y.y@nccs.com.sg

* Joint first authors
} 


\section{CLINICAL IMPACT}

\section{What is New}

- This study assesses factors influencing protective behaviours during haze episodes in Singapore.

- Knowledge of haze was associated with protective behaviours, while a lower education level and smokers were associated with lower knowledge.

- Wearing of N95 masks correlated with other protective behaviours and did not lead to a false sense of security.

\section{Clinical Implications}

- Public education remains an important public health strategy.

- Educational efforts should target those of lower education level and smokers.

- Encouraging the wearing of N95 masks can be part of a multipronged effort to minimise the impact of haze.

\section{INTRODUCTION}

Southeast Asia suffers from recurrent episodic air pollution from biomass smoke known as haze, which is mainly caused by human activities such as the extensive use of fire to clear land for agriculture, ${ }^{1}$ or to settle disputes over land rights. ${ }^{2}$ It is a major public health problem affecting an estimated 20-70 million people in the region. ${ }^{3}$ These figures are expected to increase with population growth and an increasing frequency of haze events. ${ }^{4}$

In 2013, the 3-hour Pollutant Standards Index in Singapore peaked at a hazardous level of $401 .^{5}$ Exposure to micro-particulate matter (PM), the most consistent pollutant in biomass smoke, ${ }^{6}$ is associated with adverse health effects, ranging from acute illnesses (e.g. conjunctivitis, upper respiratory tract infections) ${ }^{7}$ to exacerbations of chronic respiratory diseases (e.g. asthma, chronic obstructive pulmonary disease). ${ }^{8}$ Cardiovascular and respiratory mortality appears to increase in a concentration-dependent manner with PM exposure. ${ }^{9}$ Mild physical and psychological distress are also common among otherwise healthy individuals. ${ }^{10}$

Despite the magnitude of the problem, there is a notable absence of literature on protective behaviours during acute haze episodes, such as seeking regular updates, wearing an N95 mask, staying indoor and conducting hygience practices.
In this study, we examined the knowledge and risk perception of Singapore residents towards haze, and the protective behaviours taken. We explored the underlying associated factors that could influence an individual's protective behaviours during haze.

\section{METHODS}

\section{Study population}

This study was reported following the Strengthening the Reporting of Observational Studies in Epidemiology (STROBE) guidelines. It was a substudy of a previously published paper. The sample size was calculated based on the primary endpoint of the main study. ${ }^{11}$ We performed a cross-sectional study of a residential area in Singapore from 9 to 15 February 2015, based on a 2 -stage simple random sampling without replacement. In the first stage, 120 blocks in a public housing estate were randomly selected from 166 blocks. Subsequently, $20 \%$ of the residential units were randomly selected within each apartment block. The first individual answering the door was screened for eligibility. Inclusion criteria were English-speaking or Mandarinspeaking Singapore citizens or permanent residents, age $\geq 21$ years, residents in the estate since the 2013 haze, and the ability to wear an N95 mask independently. If that individual did not fulfil the criteria, the interviewer team would then randomly sample the next eligible resident in the household. Surveys were conducted in the evenings on weekdays and throughout the entire day on weekends. Verbal consent was obtained from all participants. An interviewer-administered questionnaire was used by the survey teams. Households with no response at the door were given a no-response notice and revisited on another occasion within the study duration. The institutional review board of the National University of Singapore granted ethical approval of the study (B-14-250).

\section{Questionnaire}

We collected demographic characteristics including age, sex, ethnicity, education, monthly household income, self-reported chronic diseases and tobacco use. Chronic disease was defined as the presence of asthma, chronic obstructive pulmonary disease or heart failure. These conditions were chosen based on the association of their exacerbations with air pollution exposure..$^{12-14}$

At the time of this study, there were no existing measures developed for understanding the knowledge, perceived risk and behaviours towards haze. We developed and validated a questionnaire to assess the 3 domains of interest (Table 1). The knowledge domain 
Table 1. Descriptive statistics of the items in knowledge, perceived risk and protective behaviours

\begin{tabular}{|c|c|}
\hline Knowledge & Correct $^{\mathrm{a}}(\%)$ \\
\hline $\mathrm{k} 1$ : Haze is caused by forest fires in neighbouring countries & 96.4 \\
\hline k2: PSI can measure severity of haze & 75.3 \\
\hline k3: Health effects of haze depends on how long one has been exposed to it & 88.2 \\
\hline $\mathrm{k} 4$ : The main pollutant during haze is particulate matter (e.g. PM10, PM2.5) & 39.1 \\
\hline $\mathrm{k} 5$ : Individuals who spend a lot of time outdoors need to be protected & 89.9 \\
\hline k6: Surgical masks provide protection from haze (reverse code) & 51.6 \\
\hline Perceived risk & High risk $^{\mathrm{b}}(\%)$ \\
\hline r1: I believe haze has a damaging effect on my health & 84.9 \\
\hline r2: I am at risk of lung disease from haze & 67.5 \\
\hline r4: I am at risk of eye disease from haze & 72.0 \\
\hline Protective behaviours & Adequate $^{c}(\%)$ \\
\hline p1: I sought updates about the severity of haze & 82.9 \\
\hline p2: I wore an N95 mask & 43.4 \\
\hline p3: I stayed indoors and avoided outdoor activities & 76.3 \\
\hline p4: I cleaned my house more frequently than usual & 57.2 \\
\hline p5: I used an air purifier & 24.3 \\
\hline p6: I took showers more frequently than usual & 40.9 \\
\hline p7: I kept myself hydrated more than usual & 76.9 \\
\hline
\end{tabular}

PSI: Pollutant Standards Index

${ }^{a}$ Original responses were dichotomised into incorrect (strongly disagree, disagree, neutral) and correct (agree, strongly agree). k6 was reversed coded prior to dichotomisation. A correct response indicates correct knowledge of the subject matter.

${ }^{\mathrm{b}}$ Original responses were dichotomised into low risk (strongly disagree, disagree, neutral) and high risk (agree, strongly agree). A high-risk response indicates a belief in haze as having a damaging effect on health as opposed to being not concerned or being unaware of the health effects of haze.

" Original responses for p1 were dichotomised into "inadequate practice" (not at all, less than weekly, weekly) and "adequate practice" (once every few days, almost every day). Adequate practice indicates that individuals seek for updates at least once every few days. Original responses for $\mathrm{p} 2$ through $\mathrm{p} 7$ were dichotomised into "inadequate practice" (not at all) and "adequate practice" (less than weekly, weekly, once every few days, almost every day).

represents general knowledge of haze and was assessed by responses to 6 factual questions on haze $(\mathrm{k} 1-\mathrm{k} 6){ }^{4,9,15}$ Perceived risk represents one's perception of personal risk of harm from haze and was assessed by responses to 4 statements $(\mathrm{r} 1-\mathrm{r} 4)$. Possible responses for these 2 domains were given on a 5-point scale, ranging from strongly disagree to strongly agree. Responses to the knowledge domain were dichotomised into correct and incorrect, and those to the perceived risk domain were dichotomised into low risk and high risk. Protective behaviours represent protective actions recommended by the National Environment Agency of Singapore that were taken during the 2013 haze and was assessed by 7 types of actions (p1-p7). A standardised visual aid with pictures of air purifiers, N95 masks and other protective behaviours was used in the interview. Possible responses were given on a 5-point scale, ranging from "not at all" to "almost every day". The responses were categorised as adequate or inadequate. The questionnaire was developed in English, translated into Chinese and back-translated to ensure semantic equivalence. Two public health experts from the Saw Swee Hock School of Public Health, National University of Singapore, verified the validity and accuracy of the questionnaire. A pilot study was conducted to test the questionnaire and survey protocols.

\section{Statistical analyses}

The analyses were performed in 2020. Demographics and survey responses were examined using frequencies 
and percentages for categorical variables, and median and range for continuous variables. Structural validity of the measurements of knowledge on haze, perceived risk and protective behaviours was tested using factor analysis, and the reliability for each construct was estimated using the McDonald's omega coefficient. ${ }^{16}$ The assessment of model goodness-of-fit followed the general criteria of $\mathrm{Hu}$ and Bentler ${ }^{17}$ and the recommendations of $\mathrm{Yu}^{18}$ for categorical outcomes (i.e. comparative fit index $\geq 0.96$, root mean square error of approximation $\leq 0.05$ ). The relationship between the demographic variables and the constructs were modelled using the structural equation modelling framework. Mplus version 8.4 (Muthén \& Muthén, Los Angeles, US) and R version 3.6.3 (R Foundation for Statistical Computing, Vienna, Austria), along with the $\mathrm{R}$ packages lavaan (version 0.6-61527) ${ }^{19}$ and semTools (version 0.5-2.921) ${ }^{20}$ were used for the analyses. All analyses were done using the weighted least square mean and variance-adjusted estimator with delta parameterisation. We obtained the bias-corrected and accelerated bootstrapped 95\% confidence interval of the parameters, based on 1,000 replications. A 2 -sided $\alpha=0.05$ was used for evaluating statistical significance.

\section{RESULTS}

\section{Participant characteristics}

A total of 2,499 households were visited by the survey teams. Of these, $541(21.6 \%)$ declined to be surveyed and 976 (39.1\%) did not respond. Among households who consented to participate, $268(10.7 \%$ of all households) did not meet the inclusion criteria and were excluded. Therefore, 714 individuals were interviewed, corresponding to a $32.0 \%$ response rate. Of these, $18(2.5 \%)$ did not complete the survey questionnaire and were excluded from the analysis. Data from the remaining 696 individuals were analysed, and their demographic characteristics are presented in Table 2. The median age of our sample was 50 years (range $21-89$ years). There were more female respondents $(53.6 \%)$. Most participants were Chinese (71.8\%). Participants with secondary school education constituted $30.3 \%$ of the sample. The most commonly reported monthly household income was USD3,500-8,400 (33.5\%). The prevalence of chronic diseases was $7.5 \%$.

\section{Descriptive statistics of knowledge, perceived risk and protective behaviours}

A total of 690 participants $(99.1 \%)$ answered at least 1 question correctly. For the knowledge measurements,
Table 2. Demographics of 696 study participants

\begin{tabular}{|c|c|}
\hline \multicolumn{2}{|l|}{ Characteristics } \\
\hline Age, median (range), year & $50(21-89)$ \\
\hline \multicolumn{2}{|l|}{ Sex, no. $(\%)$} \\
\hline Female & $373(53.6)$ \\
\hline Male & $323(46.4)$ \\
\hline \multicolumn{2}{|l|}{ Ethnicity, no. (\%) } \\
\hline Chinese & $500(71.8)$ \\
\hline Malay & $102(14.7)$ \\
\hline Indian & $86(12.4)$ \\
\hline Other & $8(1.1)$ \\
\hline \multicolumn{2}{|l|}{ Education level, no. (\%) } \\
\hline No qualification & $31(4.5)$ \\
\hline Primary school & $119(17.1)$ \\
\hline Secondary school & $211(30.3)$ \\
\hline Tertiary school (polytechnic or junior college) & $178(25.6)$ \\
\hline University & $157(22.6)$ \\
\hline \multicolumn{2}{|l|}{ Monthly household income, no. (\%) } \\
\hline No income & $139(20.0)$ \\
\hline Less than USD2100 & $100(14.4)$ \\
\hline USD2100-3500 & $166(23.9)$ \\
\hline USD3500-8400 & $233(33.5)$ \\
\hline USD8400 or more & $58(8.3)$ \\
\hline \multicolumn{2}{|l|}{ Chronic disease, no. (\%) } \\
\hline Yes & $52(7.5)$ \\
\hline No & $644(92.5)$ \\
\hline \multicolumn{2}{|l|}{ Tobacco use, no. (\%) } \\
\hline Daily & $93(13.4)$ \\
\hline Less than daily & $29(4.2)$ \\
\hline Not at all & $574(82.5)$ \\
\hline
\end{tabular}

the statement "Haze is caused by forest fires in neighbouring countries" had the highest proportion of correct responses (96.4\%); while the statement "The main pollutant during haze is particulate matter (e.g. PM10, PM2.5)" had the lowest (39.1\%). For the perceived risk measurements, most participants believed that haze had a damaging effect on their health $(84.9 \%)$. More participants felt that they were at risk for eye diseases $(72.0 \%)$ when compared with lung disease $(67.5 \%)$ or heart disease $(48.7 \%)$ (Table 1). 
For the protective behaviour measurements, the most common protective behaviours taken during the 2013 haze were seeking updates about the severity of haze through the news, Internet or radio at least once every few days $(82.9 \%)$, and staying at home and avoiding outdoor activities at least weekly (76.3\%). Fewer participants took showers more frequently than usual $(40.9 \%)$ or used air purifiers at least weekly $(24.3 \%)$. Only $43.4 \%$ of the participants wore an N95 mask (Table 1). Overall, $97.1 \%$ of participants engaged in at least 1 protective behaviour.

\section{Validation of questionnaire}

To validate the constructed measurements, a confirmatory factor analysis was first used to test the data-model fit of the respective proposed factor structure. The constructs for knowledge and protective behaviours were hypothesised to be unidimensional, and fit indices suggested satisfactory data-model fit for both single factor models. The unidimensional factor model for perceived risk did not attain a satisfactory data-model fit. Based on subsequent exploratory and confirmatory factor analysis of the perceived risk measurements, an item was removed, which resulted in an analytic-driven unidimensional model. The final unidimensional factor models showed approximately satisfactory data-model fit for each of the 3 constructs (root mean square error of approximation $\leq 0.06$; comparative fit index $\geq 0.94$ ). Point estimates of the score reliability of the measurements were all $\geq 0.56$.

\section{Mediation model}

The structural equation model is shown in Fig. 1. In general, perceived risk mediated the relationship between knowledge and protective behaviours. Protective behaviours were associated with both perceived risk $(\beta=0.28,95 \%$ confidence interval $[\mathrm{CI}]$ $0.11-0.44, P=0.002)$ and knowledge $(\beta=0.45,95 \%$ CI $0.19-0.69, P<0.001)$. Perceived risk was associated with knowledge ( $\beta=0.65,95 \%$ CI $0.48-0.79, P<0.001$ ). In addition to the direct effect from knowledge to protective behaviours, we also found a significant indirect effect from knowledge to protective behaviours $(\beta=0.18,95 \%$ CI $0.07-0.31, P=0.002)$. This indirect effect suggests that the effect of knowledge on protective behaviours is mediated by perceived risk.

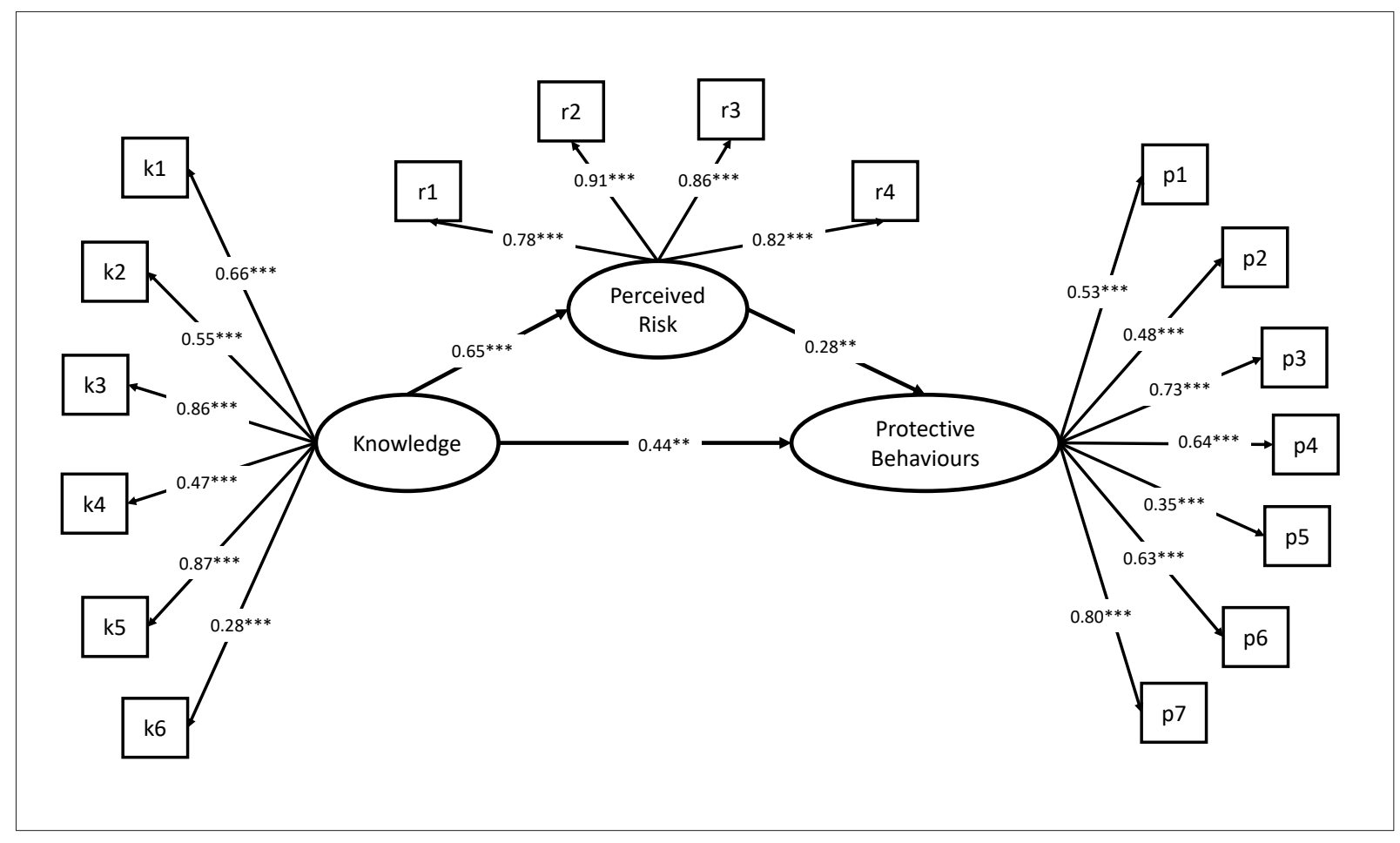

Fig. 1. Structural equation model showing the relationships among knowledge, perceived risk and protective behaviours.

The reported values are partially standardised parameter estimates (unit latent variable variance). Solid arrows indicate significant parameter estimates (e.g. from Knowledge to Perceived Risk). Control variables and demographics are omitted from the figure for brevity. Root mean square error of approximation was 0.04 and comparative fit index 0.88 . $R^{2}$ estimates of knowledge, perceived risk and protective behaviours were $0.28,0.36$ and 0.40 , respectively.

$* * P<0.01, * * * P<0.001$ 
A higher education level was associated with higher knowledge but a lower perceived risk. Smoking status was associated with lower knowledge. Malay and Indian ethnicity were associated with a lower perceived risk but higher protective behaviours. A higher household income was associated with fewer protective behaviours. Further details are described in Table 3. To examine the effect of ethnicity on the 3 constructs, we performed 2 exploratory analyses with 2 different groups (Malay and Chinese; Indian and Chinese). In both analyses, the models suggested the same underlying relationships among knowledge, perceived risk and protective behaviours.

We further classified protective behaviours as evidence-based (p1, p2, p3, p5) ${ }^{21}$ and other general recommended protective behaviours (p4, p6, p7), ${ }^{15}$ and modified the proposed mediation model. Evidencebased protective behaviours was associated with knowledge $(\beta=0.76,95 \%$ CI $0.28-0.81, P<0.001)$, while other protective behaviours was associated with perceived risk $(\beta=0.65,95 \%$ CI $0.13-0.51, P<0.001)$. Knowledge had an indirect effect on other protective behaviours mediated via perceived risk $(\beta=0.21,95 \%$ CI $0.09-0.36, P=0.002$ ). Malay and Indian ethnicity was associated only with higher other protective behaviours. A higher household income was associated with fewer other protective behaviours. Further details are described in Fig. 2 and Table 4.

\section{Association between N95 mask wearing and other protective behaviours}

We also performed analyses to assess the relationship between mask wearing and other protective behaviours. We revised the structural equation modelling and separated the item p2 "I wore an N95 mask" from other protective behaviours. The other protective behaviours were used to form a new construct called "protective behaviours (mask-unrelated)" (Fig. 3). We found a positive relationship between $\mathrm{p} 2$ and mask-unrelated protective behaviours $(\beta=0.24,95 \%$ CI $0.08-0.37$, $P=0.001)$. This relationship suggests that subjects who wore masks during the haze period tended to engage in more protective behaviours. Furthermore, we observed that knowledge affected mask wearing behaviour differently from other protective behaviours. While mask-unrelated protective behaviours was affected by knowledge both directly $(\beta=0.44,95 \%$ CI $0.18-0.69, P=0.001)$ and indirectly $(\beta=0.17,95 \%$ CI $0.06-0.30, P=0.006)$, p2 was only affected by knowledge through the indirect pathway (via perceived risk; $\beta=0.14,95 \%$ CI $0.01-0.26, P=0.034$ ) but not the direct pathway $(\beta=0.20,95 \%$ CI -0.07 to $0.43, P=0.139$ ).

\section{DISCUSSION}

In our cross-sectional study that aimed to investigate the relationships among knowledge, perceived risk and protective behaviours in response to haze, we found that protective behaviours were associated with both perceived risk and knowledge. Perceived risk was associated with knowledge, and there was an indirect effect from knowledge to protective behaviours. This indirect effect suggests that the effect of knowledge on protective behaviours is mediated by perceived risk. In addition, we found that subjects who wore masks during the haze period tended to also engage in other protective behaviours. Participants wore masks only when they perceived the haze as hazardous to their health, regardless of their knowledge level.

Despite efforts by local health authorities to educate the public through its website and radio and television broadcast to provide information about the haze, its effects on health and protective measures that one could take, ${ }^{15}$ approximately $50 \%$ of participants thought that surgical masks could provide protection and one-quarter did not know that the Pollutant Standards Index could be used to measure the severity of haze. Lower education level and smokers were associated with lower knowledge level of haze. This finding is congruent with studies demonstrating a positive association between education level and knowledge, ${ }^{22,23}$ and lower health literacy among smokers. ${ }^{24}$

Our study showed that a higher number of participants felt that their health was at risk from the haze $(84.9 \%)$, compared with a previous study on urban pollution in Los Angeles, US in the 1980s $(72.5 \%) .{ }^{25}$ Participants with higher education level, while associated with higher knowledge, had lower risk perceptions. While seemingly counterintuitive, numerous studies also found that a higher education level was associated with a lower perceived risk. ${ }^{23,26}$ Individuals with lower education levels could have lower self-efficacy and hence higher perceived risk. However, we did not examine the construct of selfefficacy. Another explanation could be poor risk perception due to lower health literacy. ${ }^{26}$ In addition, Malay and Indian ethnicity (both ethnic minorities in Singapore), compared with Chinese ethnicity, had lower risk perceptions, contrary to a previous study. ${ }^{27}$

More than $95 \%$ of our participants undertook at least 1 protective behaviour, compared with the $<50 \%$ 
Table 3. Structural equation model including knowledge on haze, perceived risk and protective behaviours ${ }^{a}$

\begin{tabular}{|c|c|c|c|}
\hline & $\beta$ & BCa $95 \%$ CI & $P$ value \\
\hline \multicolumn{4}{|l|}{ Protective behaviours predicted by } \\
\hline \multicolumn{4}{|l|}{ Knowledge } \\
\hline Direct effect & 0.45 & $0.19,0.69$ & $<0.001$ \\
\hline Indirect effect (through perceived risk) & 0.18 & $0.07,0.31$ & 0.002 \\
\hline Perceived risk & 0.28 & $0.11,0.44$ & 0.002 \\
\hline \multicolumn{4}{|l|}{ Control variables } \\
\hline Age & -0.01 & $-0.02,0.001$ & 0.084 \\
\hline Sex & 0.15 & $-0.06,0.33$ & 0.142 \\
\hline Education level & -0.12 & $-0.29,0.02$ & 0.109 \\
\hline Monthly household income & -0.10 & $-0.18,-0.02$ & 0.019 \\
\hline Chronic disease & -0.04 & $-0.44,0.31$ & 0.869 \\
\hline Smoking habit during haze & 0.02 & $-0.15,0.19$ & 0.856 \\
\hline \multicolumn{4}{|l|}{ Ethnicity } \\
\hline Chinese & [Reference] & [Reference] & [Reference] \\
\hline Malay & 0.29 & $0.004,0.56$ & 0.043 \\
\hline Indian & 0.58 & $0.26,0.92$ & 0.001 \\
\hline Others & 0.27 & $-1.17,2.18$ & 0.735 \\
\hline \multicolumn{4}{|l|}{ Perceived risk predicted by } \\
\hline Knowledge & 0.65 & $0.48,0.79$ & $<0.001$ \\
\hline \multicolumn{4}{|l|}{ Control variables } \\
\hline Age & 0.01 & $-0.002,0.02$ & 0.108 \\
\hline Sex & -0.06 & $-0.27,0.16$ & 0.558 \\
\hline Education level & -0.16 & $-0.30,-0.04$ & 0.025 \\
\hline Monthly household income & 0.02 & $-0.06,0.10$ & 0.575 \\
\hline Chronic disease & -0.02 & $-0.57,0.47$ & 0.941 \\
\hline Smoking habit during haze & -0.05 & $-0.235,0.10$ & 0.557 \\
\hline \multicolumn{4}{|l|}{ Ethnicity } \\
\hline Chinese & [Reference] & [Reference] & [Reference] \\
\hline Malay & -0.32 & $-0.64,-0.002$ & 0.052 \\
\hline Indian & -0.33 & $-0.67,-0.02$ & 0.059 \\
\hline Others & 0.23 & $-1.59,1.71$ & 0.778 \\
\hline \multicolumn{4}{|l|}{ Knowledge predicted by } \\
\hline Age & 0.001 & $-0.01,0.01$ & 0.818 \\
\hline Sex & -0.07 & $-0.26,0.16$ & 0.535 \\
\hline Education level & 0.44 & $0.33,0.56$ & $<0.001$ \\
\hline Monthly household income & 0.03 & $-0.05,0.11$ & 0.410 \\
\hline
\end{tabular}


Table 3. Structural equation model including knowledge on haze, perceived risk and protective behaviours ${ }^{\mathrm{a}}$ (Cont'd)

\begin{tabular}{lccc}
\hline & $\boldsymbol{\beta}$ & BCa 95\% CI & $\boldsymbol{P}$ value \\
\hline Chronic disease & 0.06 & $-0.45,0.61$ & 0.860 \\
\hline Smoking habit during haze & 0.18 & $0.01,0.36$ & 0.046 \\
\hline Ethnicity & & & {$[$ Reference] } \\
\hline Chinese & {$[$ Reference $]$} & {$[$ Reference] } & 0.425 \\
Malay & -0.13 & $-0.44,0.20$ & 0.891 \\
\hline Indian & 0.03 & $-0.35,0.51$ & 0.324 \\
\hline Others & -1.03 & $-1.99,1.44$ & \\
\hline
\end{tabular}

BCa: bias-corrected and accelerated; CI: confidence interval

${ }^{a} \beta$ refers to partially standardised regression weights (unit latent variable variance). Root mean square error of approximation was 0.04 and comparative fit index 0.88 . Wald test showed a significant overall effect of ethnicity on protective behaviours $\left(W_{T}(3)=17.36, P<0.001\right)$ and perceived risk $\left(W_{T}(3)=8.92, P=0.030\right)$, but not on knowledge $\left(W_{T}(3)=4.56, P=0.207\right)$. Coding for variables: sex ( 0 : male; 1 : female), chronic disease ( 0 : no; 1 : yes) and smoking habit (1: daily; 2 : less than daily; 3 : not at all).

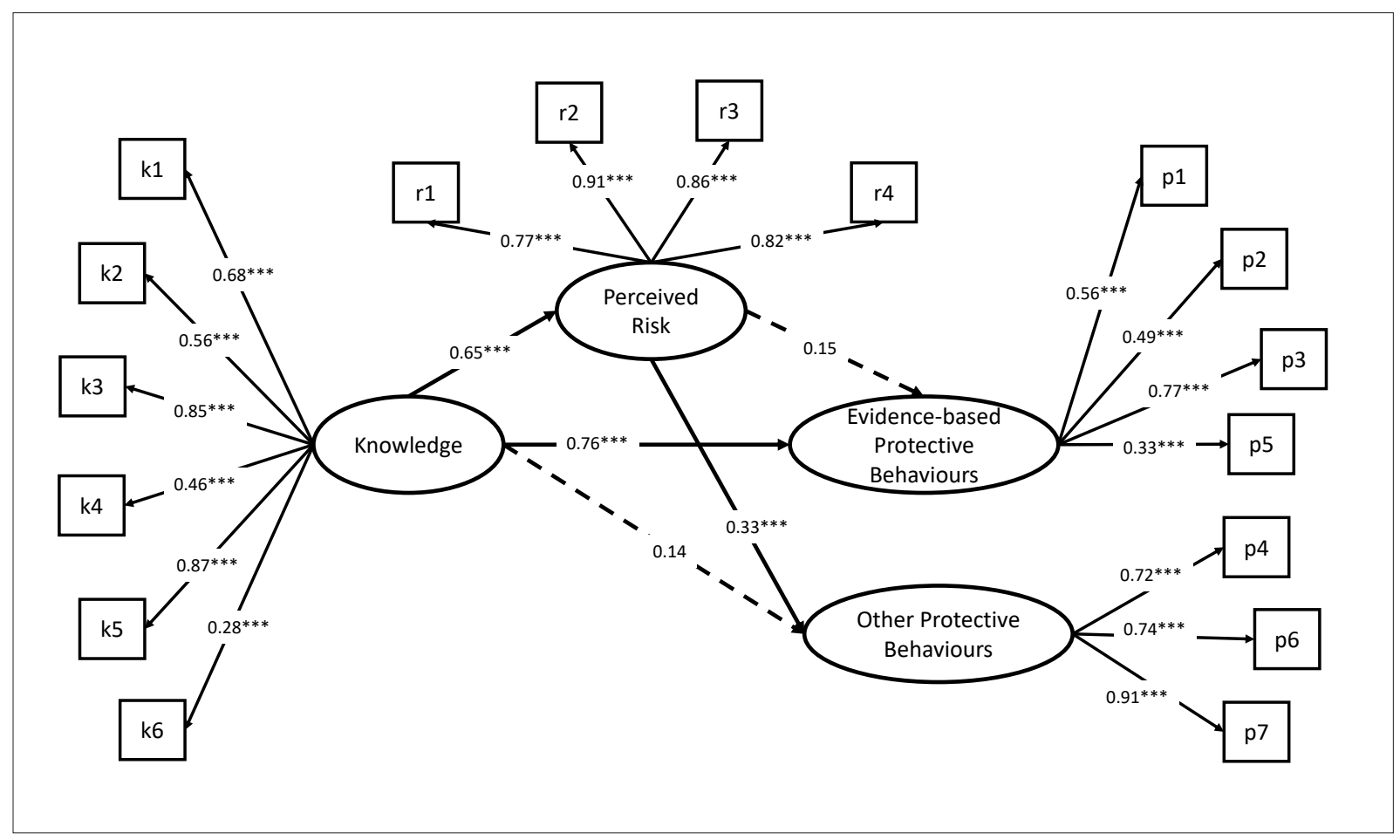

Fig. 2. Structural equation model showing the relationships among knowledge, perceived risk and evidence-based and other protective behaviours.

The reported values are partially standardised parameter estimates (unit latent variable variance). Solid arrows indicate significant parameter estimates (e.g. from Knowledge to Perceived Risk) whereas dashed arrows indicate non-significant parameter estimates (e.g. from Perceived Risk to Evidence-based Protective Behaviours). Control variables and demographics are omitted from the figure for brevity. Root mean square error of approximation was 0.04 and comparative fit index 0.92 . $R^{2}$ estimates of knowledge, perceived risk, evidence-based protective behaviours and other protective behaviour were $0.28,0.35,0.64$ and 0.24 , respectively. $* * * P<0.001$

uptake of health advisory recommended measures against urban air pollution. ${ }^{25,28}$ This finding may reflect the severity of the 2013 haze episode. Alternatively, it suggests a decline in the perceived risk and practice of personal protective behaviours as people grow accustomed to constant urban air pollution, compared with acute episodes of haze. ${ }^{29}$ There is a strong association between knowledge and protective behaviours, similar to that observed in urban air pollution $^{25}$ and influenza. ${ }^{27,30}$ While there was a 
Table 4. Structural equation model including knowledge on haze, perceived risk, and evidence-based and other protective behaviours ${ }^{\mathrm{a}}$

\begin{tabular}{|c|c|c|c|}
\hline & $\beta$ & BCa 95\% CI & $P$ value \\
\hline \multicolumn{4}{|c|}{ Evidence-based protective behaviours predicted by } \\
\hline \multicolumn{4}{|l|}{ Knowledge } \\
\hline Direct effect & 0.76 & $0.28,0.81$ & $<0.001$ \\
\hline Indirect effect (through perceived risk) & 0.10 & $-0.03,0.15$ & 0.179 \\
\hline Perceived risk & 0.15 & $-0.07,0.37$ & 0.170 \\
\hline \multicolumn{4}{|l|}{ Control variables } \\
\hline Age & -0.001 & $-0.01,0.01$ & 0.835 \\
\hline Sex & 0.20 & $-0.04,0.47$ & 0.121 \\
\hline Education level & -0.12 & $-0.32,0.05$ & 0.197 \\
\hline Monthly household income & -0.06 & $-0.16,0.03$ & 0.214 \\
\hline Chronic disease & 0.28 & $-0.27,0.83$ & 0.382 \\
\hline Smoking habit during haze & -0.01 & $-0.23,0.18$ & 0.901 \\
\hline \multicolumn{4}{|l|}{ Ethnicity } \\
\hline Chinese & [Reference] & [Reference] & [Reference] \\
\hline Malay & 0.24 & $-0.15,0.58$ & 0.222 \\
\hline Indian & 0.19 & $-0.19,0.63$ & 0.387 \\
\hline Others & 0.33 & $-1.82,2.72$ & 0.750 \\
\hline
\end{tabular}

Other protective behaviours predicted by

\begin{tabular}{|c|c|c|c|}
\hline \multicolumn{4}{|l|}{ Knowledge } \\
\hline Direct effect & 0.14 & $-0.14,0.39$ & 0.302 \\
\hline Indirect effect (through perceived risk) & 0.21 & $0.09,0.36$ & 0.002 \\
\hline Perceived risk & 0.65 & $0.13,0.51$ & $<0.001$ \\
\hline \multicolumn{4}{|l|}{ Control Variables } \\
\hline Age & -0.01 & $-0.02,-0.002$ & 0.016 \\
\hline Sex & 0.08 & $-0.12,0.27$ & 0.422 \\
\hline Education level & -0.10 & $-0.24,0.07$ & 0.234 \\
\hline Monthly household income & -0.10 & $-0.18,-0.02$ & 0.010 \\
\hline Chronic disease & -0.22 & $-0.57,0.12$ & 0.228 \\
\hline Smoking habit during haze & 0.04 & $-0.12,0.21$ & 0.654 \\
\hline \multicolumn{4}{|l|}{ Ethnicity } \\
\hline Chinese & [Reference] & [Reference] & [Reference] \\
\hline Malay & 0.28 & $0.01,0.57$ & 0.044 \\
\hline Indian & 0.77 & $0.48,1.10$ & $<0.001$ \\
\hline Other & 0.27 & $-0.94,2.58$ & 0.735 \\
\hline \multicolumn{4}{|l|}{ Perceived risk predicted by } \\
\hline Knowledge & 0.65 & $0.48,0.79$ & $<0.001$ \\
\hline
\end{tabular}


Table 4. Structural equation model including knowledge on haze, perceived risk, and evidence-based and other protective behaviours ${ }^{\mathrm{a}}$ (Cont'd)

\begin{tabular}{|c|c|c|c|}
\hline & $\beta$ & BCa $95 \%$ CI & $P$ value \\
\hline \multicolumn{4}{|l|}{ Control variables } \\
\hline Age & 0.01 & $-0.002,0.02$ & 0.104 \\
\hline Sex & -0.07 & $-0.28,0.16$ & 0.539 \\
\hline Education Level & -0.16 & $-0.30,-0.04$ & 0.025 \\
\hline Monthly household income & 0.02 & $-0.06,0.10$ & 0.583 \\
\hline Chronic disease & -0.02 & $-0.57,0.47$ & 0.942 \\
\hline Smoking habit during haze & -0.05 & $-0.25,0.10$ & 0.569 \\
\hline \multicolumn{4}{|l|}{ Ethnicity } \\
\hline Chinese & [Reference] & [Reference] & [Reference] \\
\hline Indian & -0.33 & $-0.68,-0.02$ & 0.060 \\
\hline Others & 0.23 & $-1.56,1.73$ & 0.773 \\
\hline \multicolumn{4}{|l|}{ Knowledge predicted by } \\
\hline Age & 0.001 & $-0.01,0.01$ & 0.837 \\
\hline Sex & -0.06 & $-0.26,0.16$ & 0.566 \\
\hline Education level & 0.44 & $0.33,0.56$ & $<0.001$ \\
\hline Monthly household income & 0.03 & $-0.05,0.11$ & 0.400 \\
\hline Chronic disease & 0.06 & $-0.41,0.62$ & 0.862 \\
\hline Smoking habit during haze & 0.18 & $0.01,0.37$ & 0.049 \\
\hline Chinese & [Reference] & [Reference] & [Reference] \\
\hline Malay & -0.13 & $-0.44,0.21$ & 0.437 \\
\hline Indian & 0.03 & $-0.35,0.51$ & 0.884 \\
\hline Other & -1.04 & $-2.08,1.34$ & 0.321 \\
\hline
\end{tabular}

BCa: bias-corrected and accelerated; CI: confidence interval

${ }^{a} \beta$ refers to partially-standardised regression weights (unit latent variable variance). Root mean square error of approximation was 0.04 and comparative fit index 0.92. Coding for variables: sex (0: male; 1 : female), chronic disease (0: no; 1 : yes) and smoking habit (1: daily; 2 : less than daily; 3 : not at all).

direct effect between knowledge and evidence-based protective behaviours, the association between knowledge and other protective behaviours was fully mediated by perceived risk. Perceived risk was associated with the overall protective behaviours. This provides partial empirical evidence for the health belief model, which proposes that a higher perceived risk will result in a higher likelihood of protective behaviour. ${ }^{31}$

In our study, we also found that household income levels were inversely associated with other protective behaviours, while there was no association between household income and evidence-based protective behaviours. These results have not been found in previous studies in urban air pollution ${ }^{32}$ or influenza, ${ }^{27}$ and run contrary to the well demonstrated inverse relationship between socioeconomic status and unhealthy behaviours. ${ }^{33} \mathrm{~A}$ possible explanation might be that individuals of lower socioeconomic status are more likely to take public transport and be involved in non-office-based occupations, and be more exposed to the negative effects of haze. Hence, they may see a greater need for protective behaviours. In addition, the negative effect of income disparity could have been mitigated by the targeted effort by the Singapore government and multiple voluntary welfare organisation 


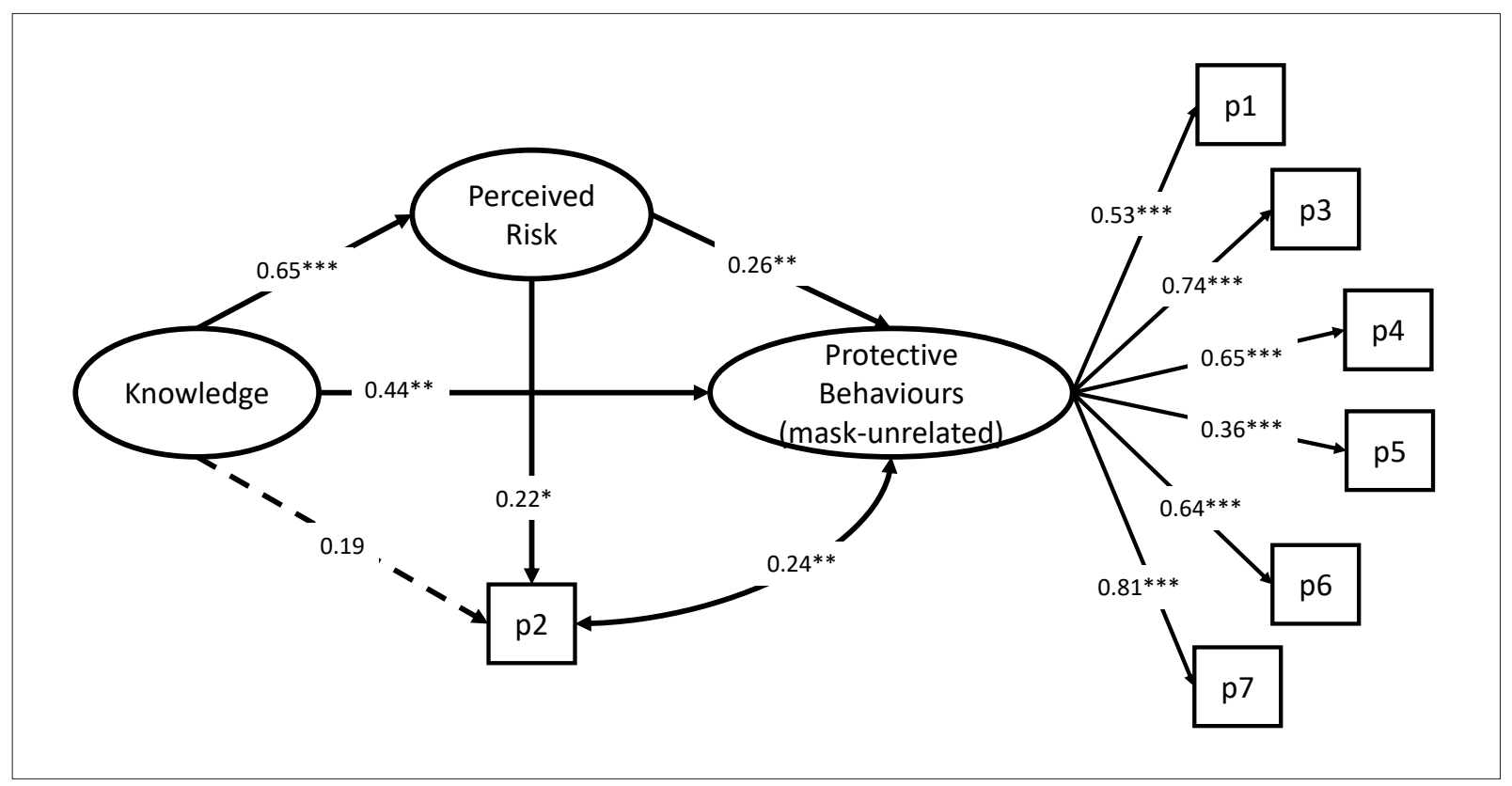

Fig. 3. Structural equation model showing the relationships among knowledge, perceived risk, mask-unrelated protective behaviours and p2 ("I wore an N95 mask").

The reported values are partially standardised parameter estimates (unit latent variable variance). Solid arrows indicate significant parameter estimates (e.g. from Knowledge to Perceived Risk) whereas dashed arrows indicate non-significant parameter estimates (e.g. from Knowledge to p2). Unidirectional arrows (e.g. from Knowledge to Perceived Risk) indicates that Perceived Risk was regressed on Knowledge, whereas the bidirectional arrow indicates correlational relationship between constructs (e.g. between error variances of Protective Behaviours (mask-unrelated) and p2). Control variables and indicators of Knowledge and Perceived Risk are omitted from the figure. Root mean square error of approximation was 0.04 and comparative fit index 0.89 .

$* P<0.05, * * P<0.01, * * * P<0.001$

that distributed N95 masks and household essentials to residents with lower household income. ${ }^{34}$

In addition, ethnic minorities were more likely to adopt other protective behaviours. A previous study suggested a shared perceived vulnerability. ${ }^{27}$ However, our study showed that perceived risks were also lower in these groups as well, despite a higher frequency of other protective behaviours. Exploratory analyses with each ethnic group suggested the same underlying relationships among knowledge, perceived risk and protective behaviours, suggesting that reasons beyond the existing constructs may explain the seemingly contradictory relationships. More research will need to be conducted to elucidate the relationship between ethnicity and protective behaviours.

Together, our results suggest the importance of increasing knowledge of haze, as a higher knowledge level was associated with greater frequency of protective behaviours. While the public education efforts by the Singapore government were commendable, there can be significant room for improvement as there were significant knowledge gaps among the participants. Educational effort should be directed at smokers and those with a lower educational level.
While N95 masks are an effective means of reducing exposure to PM, only $43.4 \%$ of participants reported using them during the haze. In addition, their efficacy was dependent on proper fitting. A previous study found that only $12.6 \%$ of participants in the community could don the N95 mask proficiently. ${ }^{11}$ There were concerns that poorly fitted N95 mask may create a false sense of security and lead to lower adoption of other protective behaviours (e.g. staying indoors). In our analysis, we found that wearing of the N95 mask was associated with other mask-unrelated protective behaviours and there was no evidence of poor adoption of other protective behaviours.

The strength of this study was the relatively large sample size. The use of a structural equation modelling allowed us to elucidate complex interactions among demographic characteristics, knowledge, perceived risk and protective behaviours. In addition, good fit indices and good internal consistencies of our subscales improved the credibility of our findings.

Our study, however, had several limitations. This was a cross-sectional study and the temporal relationship between the constructs could not be assessed. The response rate of $32.0 \%$ might have introduced non- 
response bias. Also, there could be recall bias. We examined the relationship between the knowledge at the time of survey and protective behaviours during the haze. It was possible that participants had become more knowledgeable after experiencing the severe 2013 haze episode. This study was conducted in a public housing estate and omitted the high-income earners, possibly explaining the contradictory finding of the inverse relationship between income and other protective behaviours. However, the majority of Singapore citizens and permanent residents $(>80 \%)$ live in public housing and the study would be generalisable to them. The questionnaire was administered in only English and Chinese. This limitation might have excluded ethnic minority participants who were non-native speakers of the 2 languages. However, the ethnic composition of our participants was largely consistent with the Singapore Census data in 2015 (Chinese $74.3 \%$, Malay $13.3 \%$, Indian $9.1 \%$ and others $3.2 \%$ ), which suggested that ethnic minorities were not underrepresented in our study. ${ }^{35}$ Another limitation was the significant time interval between data collection and analysis (5-year gap). However, insights gained in this study to encourage the adoption of protective behaviours are likely to be applicable to other urban populations facing the issue of haze and perhaps even infectious disease outbreaks (e.g. COVID-19 pandemic), given the many similarities between these 2 issues and the protective behaviours required. The COVID-19 pandemic demonstrated the importance of public education to counter disinformation and to increase uptake of protective behaviours.

The nature of the haze in Southeast Asia and its associated health consequences present a unique and recurrent public health problem. The link between haze and acute adverse health outcomes has been well studied; ${ }^{7,8,36}$ hence, encouraging protective behaviours is a logical solution. Our findings suggest that knowledge about the haze is directly associated with the practice of protective behaviours, the association of which is also mediated via perceived risk. Interventions that increase knowledge, like public education, remain a pertinent public health strategy. In addition, the wearing of N95 masks correlates with uptake of other protective behaviours and can be part of the multipronged effort to minimise the impact of haze, provided proper fitting and training are performed.

\section{Disclosure}

Dr Bee Choo Tai reported receiving personal fees from Boehringer Ingelheim and Wiley-Blackwell outside the submitted work.

\section{Acknowledgements}

The authors thank the following individuals for providing help in this study: Abigail Lee, Alexandra Yung, Bing Howe Lee, Bochao Jiang, Cheryl Lam, Deborah Lim, Fiona Ng, George Tan, Jasmine Chang, Jiaquan Chaung, Jia Long Chua, Kevin Lim, Kristabella Low, Lorraine Yong, Lyria Hoa, Marcus Tan, Marianne Tsang, Michael Chee, Nicholas Ngiam, Nicole Chan, Nicole Chew, Ren Wei Liu, Shaina Neo and Zhao Kai Low. The authors also thank Professor Sin Eng Chia for providing guidance.

\section{REFERENCES}

1. Sastry N. Forest fires, air pollution, and mortality in Southeast Asia. Demography 2002;39:1-23.

2. Moore PF, Ganz D, Tan LC, et al. Communities in Flames: Proceedings of an International Conference on Community Involvement in Fire Management. Bangkok, Thailand: FAO Regional Office for Asia and the Pacific; 2002.

3. Steffen W, Jäger J, Carson DJ, Bradshaw C (Eds). Challenges of a Changing Earth: Proceedings of the Global Change Open Science Conference, Amsterdam, The Netherlands, 10-13 July 2001. Berlin: Springer-Verlag; 2002.

4. Gaveau DLA, Salim MA, Hergoualc'h K, et al. Major atmospheric emissions from peat fires in Southeast Asia during non-drought years: evidence from the 2013 Sumatran fires. Sci Rep 2014;4:6112.

5. The Straits Times. Haze update: PSI 401 at noon; many pharmacies still out of masks, 21 June 2013. Available at: https://www.straitstimes. com/singapore/haze-update-psi-401-at-noon-many-pharmacies-stillout-of-masks. Accessed on 20 April 2020.

6. Levine JS. The 1997 fires in Kalimantan and Sumatra, Indonesia: gaseous and particulate emissions. Geophys Res Lett 1999;26:815-8.

7. Wiwatanadate P. Acute air pollution-related symptoms among residents in Chiang Mai, Thailand. J Environ Health 2014;76:76-84.

8. Emmanuel SC. Impact to lung health of haze from forest fires: the Singapore experience. Respirology 2000;5:175-82.

9. Samoli E, Peng R, Ramsay T, et al. Acute effects of ambient particulate matter on mortality in Europe and North America: results from the APHENA study. Environ Health Perspect 2008; 116:1480-6.

10. Ho RC, Zhang MW, Ho CS, et al. Impact of 2013 south Asian haze crisis: study of physical and psychological symptoms and perceived dangerousness of pollution level. BMC Psychiatry 2014;14:81.

11. Yeung W, $\mathrm{Ng} \mathrm{K}$, Fong JMN, et al. Assessment of proficiency of N95 mask donning among the general public in Singapore. JAMA Netw Open 2020;3:e209670.

12. Shah ASV, Langrish JP, Nair H, et al. Global association of air pollution and heart failure: a systematic review and meta-analysis. Lancet 2013;382:1039-48

13. Mott JA, Mannino DM, Alverson CJ, et al. Cardiorespiratory hospitalizations associated with smoke exposure during the 1997, Southeast Asian forest fires. Int J Hyg Environ Health 2005; 208:75-85.

14. Ko FW, Hui DS. Air pollution and chronic obstructive pulmonary disease. Respirology 2012;17:395-401.

15. National Environment Agency. Air pollution FAQs. Available at: https://www.nea.gov.sg/our-services/pollution-control/air-pollution/ faqs. Accessed on 20 April 2020.

16. McDonald RP. Test Theory: A Unified Treatment. New Jersey: Lawrence Erlbaum Associates; 1999. 
17. Hu LT, Bentler PM. Cutoff criteria for fit indexes in covariance structure analysis: conventional criteria versus new alternatives. Struct Equ Modeling 1999;6:1-55.

18. Yu CY. Evaluating Cutoff Criteria of Model Fit Indices for Latent Variable Models With Binary and Continuous Outcomes [dissertation]. Los Angeles: University of California; 2002.

19. Rosseel Y. lavaan: an $\mathrm{R}$ package for structural equation modeling. J Stat Softw 2012;48:1-36

20. Jorgensen TD, Pornprasertmanit S, Schoemann AM, et al. semTools: Useful tools for structural equation modeling. $\mathrm{R}$ package version 0.5-2.9212020. Available at https://CRAN.R-project.org/ package $=$ semTools. Accessed on 20 April 2020

21. Carlsten C, Salvi S, Wong GWK, et al. Personal strategies to minimise effects of air pollution on respiratory health: advice for providers, patients and the public. Eur Respir J 2020;55:1902056.

22. Wang R, Yang Y, Chen R, et al. Knowledge, attitudes, and practices (KAP) of the relationship between air pollution and children's respiratory health in Shanghai, China. Int J Environ Res Public Health 2015;12:1834-48.

23. Qian $\mathrm{X}, \mathrm{Xu} \mathrm{G}, \mathrm{Li} \mathrm{L}$, et al. Knowledge and perceptions of air pollution in Ningbo, China. BMC Public Health 2016;16:1138.

24. Fawns-Ritchie C, Starr JM, Deary IJ. Health literacy, cognitive ability and smoking: a cross-sectional analysis of the English Longitudinal Study of Ageing. BMJ Open 2018;8:e023929.

25. Evans GW, Colome SD, Shearer DF. Psychological reactions to air pollution. Environ Res 1988;45:1-15.

26. Gidengil CA, Parker AM, Zikmund-Fisher BJ. Trends in risk perceptions and vaccination intentions: a longitudinal study of the first year of the H1N1 pandemic. Am J Public Health 2012;102:672-9.
27. Yap J, Lee VJ, Yau TY, et al. Knowledge, attitudes and practices towards pandemic influenza among cases, close contacts, and healthcare workers in tropical Singapore: a cross-sectional survey. BMC Public Health 2010;10:442.

28. Skov T, Cordtz T, Jensen LK, et al. Modifications of health behaviour in response to air pollution notifications in Copenhagen. Soc Sci Med 1991;33:621-6.

29. Slovic P, Peters E, Finucane ML, et al. Affect, risk, and decision making. Health Psychol 2005;24(4 Suppl):S35-40.

30. Kim Y, Zhong W, Jehn M, et al. Public risk perceptions and preventive behaviors during the $2009 \mathrm{H} 1 \mathrm{~N} 1$ influenza pandemic. Disaster Med Public Health Prep 2015;9:145-54.

31. Janz NK, Becker MH. The Health Belief Model: a decade later. Health Educ Q 1984;11:1-47.

32. Oltra C, Sala R. Perception of risk from air pollution and reported behaviors: a cross-sectional survey study in four cities. J Risk Res 2018;21:869-84

33. Pampel FC, Krueger PM, Denney JT. Socioeconomic disparities in health behaviors. Annu Rev Sociol 2010;36:349-70.

34. The Straits Times. MOH pushes out four million masks from government stockpile, 24 June 2013. Available at: https://www. straitstimes.com/singapore/moh-pushes-out-four-million-masks-fromgovernment-stockpile. Accessed on 20 April 2020.

35. Singapore Department of Statistics. General Household Survey 2015, March 2016. Available at: https://www.singstat.gov.sg/publications/ ghs/ghs2015. Accessed on 20 April 2020.

36. Afroz R, Hassan MN, Ibrahim NA. Review of air pollution and health impacts in Malaysia. Environ Res 2003;92:71-7. 